\title{
Phase II study of docetaxel, oxaliplatin, and S-1 therapy in patients with metastatic gastric cancer
}

\author{
Hyeong Su Kim ${ }^{1} \cdot$ Min-Hee Ryu ${ }^{2} \cdot$ Dae Young Zang ${ }^{1} \cdot$ Baek-Yeol Ryoo $^{2}$ • \\ Dae Hyun Yang ${ }^{3} \cdot \mathrm{Ji}$ Woong $\mathrm{Cho}^{3} \cdot$ Man Sup $\mathrm{Lim}^{3} \cdot$ Min-Jeong Kim ${ }^{4}$. \\ Boram Han ${ }^{1} \cdot$ Dae Ro Choi ${ }^{1} \cdot$ Jung Han Kim ${ }^{1} \cdot$ Joo Young Jung ${ }^{1}$. \\ Hunho Song ${ }^{1}$ - Choong Kee Park ${ }^{1}$ - Yoon-Koo Kang ${ }^{2}$
}

Received: 29 January 2015/ Accepted: 29 April 2015/Published online: 22 May 2015

(c) The International Gastric Cancer Association and The Japanese Gastric Cancer Association 2015

\begin{abstract}
Background Although the docetaxel, 5-fluorouracil, and cisplatin triplet has yielded significant improvements in time to progression, overall survival, and overall response rate, the high incidence of severe adverse events limits the use of the docetaxel, 5-fluorouracil, and cisplatin triplet. To overcome this limitation, we evaluated the efficacy and safety of the combination of docetaxel, oxaliplatin, and S-1 for the treatment of metastatic gastric cancer.

Methods Chemotherapy-naive patients with pathologically proven unresectable recurrent or metastatic gastric adenocarcinoma were assessed for eligibility. Docetaxel at $52.5 \mathrm{mg} / \mathrm{m}^{2}$ and oxaliplatin at $105 \mathrm{mg} / \mathrm{m}^{2}$ were administered intravenously on day 1 , and S-1 was administered orally at $80 \mathrm{mg} / \mathrm{m}^{2}$ on days $1-14$ of every 21-day cycle.
\end{abstract}

ClinicalTrials.gov identifier NCT00525005

H. S. Kim and M.-H. Ryu contributed equally to this study.

Dae Young Zang

fhdzang@hallym.ac.kr

1 Division of Hematology-Oncology, Department of Internal Medicine, Hallym University Medical Center, Hallym University College of Medicine, 22, Gwanpyeong-ro 170 beon-gil, Dongan-gu, Anyang 431-796, South Korea

2 Department of Oncology, Asan Medical Center, University of Ulsan College of Medicine, Seoul, South Korea

3 Department of Surgery, Hallym University Medical Center, Hallym University College of Medicine, Anyang, South Korea

4 Department of Radiology, Hallym University Medical Center, Hallym University College of Medicine, Anyang, South Korea
Results Forty-four patients (median age 54.5 years) were enrolled. All patients had metastatic disease. A total of 340 cycles of chemotherapy were administered (median of eight cycles per patient; range 1-36 cycles). Toxicities were evaluated in 43 patients, and the responses were evaluated in 40 patients. Major toxicities included grade $3 / 4$ neutropenia $(37.2 \%)$ and leukopenia $(27.9 \%)$. The overall response rate was $54.5 \%$ [95\% confidence interval (CI) $40.1-68.3 \%$ ] in the intention-to-treat population. The median progression-free survival and overall survival were 7.6 months (95\% CI 6.2-9.0 months) and 12.0 months (95\% CI 6.9-17.2 months), respectively.

Conclusion These data suggest that the docetaxel, oxaliplatin, and S-1 combination regimen is effective and relatively well tolerable, and it seems to have potential to be a reasonable therapeutic strategy in patients with metastatic or recurrent gastric cancer.

Keywords Stomach neoplasm - Docetaxel - Oxaliplatin . S-1 · Phase II clinical trial

\section{Introduction}

Gastric cancer is the third commonest cause of cancer-driven death worldwide [1]. Many patients present initially with locally advanced disease or distant metastases, and a considerable number of patients develop recurrence even after complete resection [2]. For these patients, combination chemotherapy has been shown to improve the quality of life and overall survival (OS) compared with best supportive care alone [3]. Although a standard front-line chemotherapy regimen for advanced gastric cancer (AGC) has not yet been established, the combination of epirubicin, cisplatin or oxaliplatin, and fluoropyrimidine has been considered a 
reference regimen in Europe. In other regions, platinum and fluoropyrimidine doublets have been adopted as a reference regimen in clinical trials. In a phase III trial, the docetaxel, 5-fluorouracil (5-FU), and cisplatin (DCF) triplet yielded significant improvements in time to progression (TTP), OS, and overall response rate (ORR) compared with the cisplatin and 5-FU doublet in AGC [4]. Nevertheless, the high incidence of severe adverse events such as febrile neutropenia and infection and the inconvenience of continuous intravenous 5-FU infusion limit the use of this regimen worldwide as a popular chemotherapeutic regimen.

Docetaxel is an active agent for treating patients with gastric cancer, with ORRs of $16-24 \%$ as a single agent [5-7] and 19-37\% in combination with 5-FU and/or cisplatin $[4,8,9]$. Oxaliplatin is a platinum analog with less nephrotoxicity than cisplatin, and its efficacy is not inferior to that of cisplatin in the treatment of AGC [10, 11]. S-1, an oral 5-FU prodrug, is active against AGC as a single agent or in combination with cisplatin in phase III trials. These trials suggest that S-1 was not inferior to 5-FU, with less toxicity, and can replace 5-FU for the treatment of AGC $[12,13]$. The rationale for the combination of docetaxel, oxaliplatin, and S-1 (DOS) is that each agent is active against gastric cancer with a distinct mechanism of action and the principal toxicities of these drugs are different: neutropenia is the principal toxicity of docetaxel, neuropathy is the major toxicity of oxaliplatin, and gastrointestinal toxicity is the main side effect of S-1.

Considering the evident efficacies and more favorable toxicity profiles in addition to the more convenient administration of oxaliplatin and S-1 (SOX) compared with cisplatin and infusional 5-FU, the DOS regimen was expected to be better tolerated, more convenient, and more effective than previously used therapies. We performed a dose-finding study using the DOS regimen [14]. The doselimiting toxicity was neutropenia, and the ORR was $67 \%$. The recommended DOS chemotherapy in patients with AGC was docetaxel at $52.5 \mathrm{mg} / \mathrm{m}^{2}$ and oxaliplatin at $105 \mathrm{mg} / \mathrm{m}^{2}$ on day 1 , and $\mathrm{S}-1$ at $80 \mathrm{mg} / \mathrm{m}^{2}$ on days $1-14$ of every 21-day cycle.

Therefore, we conducted a phase II study to investigate the efficacy and feasibility of the DOS regimen in patients with AGC.

\section{Patients and methods}

\section{Study design}

This trial was a single-arm phase II study evaluating combination chemotherapy with DOS. The primary end point was ORR, and the secondary end points were OS, progression-free survival (PFS), and assessment of toxicity.
The investigation was performed in accordance with the Declaration of Helsinki, and the protocol was approved by the institutional review boards of Hallym University Medical Center, Anyang, South Korea and Asan Medical Center, Seoul, South Korea.

\section{Eligibility}

Patients with pathologically proven unresectable recurrent or metastatic gastric adenocarcinoma were assessed for eligibility. The major inclusion criteria were as follows: measurable disease based on Response Evaluation Criteria in Solid Tumors (RECIST) version 1.0 [15]; age 18-70 years; an Eastern Cooperative Oncology Group (ECOG) performance status (PS) of $0-2$; adequate bone marrow function (defined by a leukocyte count of $4000 / \mu \mathrm{L}$ or greater, an absolute neutrophil count of $1500 / \mu \mathrm{L}$ or greater, a platelet count of $100,000 / \mu \mathrm{L}$ or greater, and a serum hemoglobin level of $9 \mathrm{~g} / \mathrm{dL}$ or greater); adequate renal function (serum creatinine level below $1.5 \mathrm{mg} / \mathrm{dL}$ ); and adequate hepatic function (bilirubin level below $2.0 \mathrm{mg} / \mathrm{dL}$, and aspartate aminotransferase and alanine aminotransferase levels twofold or less of the reference value). Prior radiotherapy was permitted if it was not administered to the target lesions evaluated in this study and if it had been completed at least 4 weeks before enrollment of the patient into the study.

Patients in whom adjuvant chemotherapy had been completed at least 6 months before recruitment were enrolled. However, patients with recurrence after adjuvant S-1-, oxaliplatin-, or docetaxel-based chemotherapy were not eligible for the study regardless of the time to recurrence. Patients with brain metastasis, obvious bowel obstruction, or serious gastrointestinal bleeding were excluded. Each patient provided written informed consent before study enrollment.

\section{Pretreatment evaluations}

Baseline evaluations included confirmation of the pathologic diagnosis, medical history, physical examination, ECOG PS, complete blood count, serum chemistry and electrolytes, urine analysis, chest X-ray, and three-dimensional computed tomography.

\section{Treatment schedule}

The treatment consisted of intravenous administration of docetaxel $\left(\right.$ Taxotere $^{\circledR}$, Sanofi-Aventis, Paris, France) at $52.5 \mathrm{mg} / \mathrm{m}^{2}$ mixed with $200 \mathrm{~mL}$ of $5 \%$ dextrose solution in $60 \mathrm{~min}$ on day 1 , followed by intravenous administration of oxaliplatin (Eloxatin ${ }^{\circledR}$, Sanofi-Aventis, Paris, France) at $105 \mathrm{mg} / \mathrm{m}^{2}$ mixed in $250 \mathrm{~mL}$ of $5 \%$ dextrose solution in 
$2 \mathrm{~h}$ on day 1 and orally administered S- 1 (TS- $1^{\circledR}$, Taiho, Tokyo, Japan) at $40 \mathrm{mg} / \mathrm{m}^{2}$ twice daily on days $1-14$ of each cycle. Patients with a body surface area of less than $1.25 \mathrm{~m}^{2}$ received $80 \mathrm{mg} \mathrm{S}-1$ daily, those with a body surface area of $1.25 \mathrm{~m}^{2}$ or more but less than $1.5 \mathrm{~m}^{2}$ received $100 \mathrm{mg} \mathrm{S}-1$ daily, and those with a body surface area of $1.5 \mathrm{~m}^{2}$ or more received $120 \mathrm{mg} \mathrm{S}-1$ daily. Prophylactic dexamethasone was prescribed to prevent potential hypersensitivity reactions to docetaxel. The treatment was repeated every 3 weeks until there was disease progression or unacceptable toxicity or the patient withdrew consent. Standard antiemetic prophylaxis by intravenously or orally administered $5-\mathrm{HT}_{3}$ antagonists was administered before and after chemotherapy. Granulocyte colony stimulating factor was administered to treat neutropenic events; however, prophylactic granulocyte colony stimulating factor and prophylactic antibiotics were not allowed in patients who had experienced a neutropenic event in the previous cycle.

\section{Dose modifications}

Dose modifications were done according to the study protocol. The next treatment cycle was initiated only when the neutrophil count was $1500 / \mu \mathrm{L}$ or greater and the platelet count was $100,000 / \mu \mathrm{L}$ or greater. Treatment was delayed in the event of grade 3/4 nonhematologic toxicities until the toxicities were resolved to grade 1 or lower. The doses of docetaxel and oxaliplatin were reduced by $25 \%$ of the initial doses for related grade 3 toxicity or for the second occurrence of the same grade 2 toxicity. The dosage of S-1 was reduced by $20 \mathrm{mg} /$ day for related grade 3 toxic effects or for the second occurrence of the same grade 2 toxicity. The doses of docetaxel and oxaliplatin were reduced by $50 \%$ of the initial dose for related grade 4 toxicity, for the second occurrence of the same grade 3 toxicity, or for the third occurrence of same grade 2 toxicity. The dosage of S-1 was reduced by $40 \mathrm{mg} / \mathrm{day}$ for related grade 4 toxicity, for the second occurrence of the same grade 3 toxicity, or for the third occurrence of the same grade 2 toxicity. Treatment was discontinued if, despite the dose reduction, the same toxicity occurred for a fourth time at grade 2, a third time at grade 3, or a second time at grade 4 . In addition, if the toxicity had not improved to grade 0 or grade 1 after 3 weeks, the patient was removed from the study. The dose reduction was maintained in subsequent cycles.

\section{Response and toxicity evaluation}

The response to therapy was assessed by radiological evaluation of any measurable lesion every two cycles based on RECIST version 1.0 [15] using three-dimensional computed tomography, and was determined by an independent response review committee. Confirmation of all complete and partial responses was required 4 weeks later. After discontinuation of the study treatment, patients were followed up every 3 months until disease progression or death.

Toxicity was evaluated and recorded according to version 3.0 of the Common Terminology Criteria for Adverse Events of the National Cancer Institute. All of the patients who received at least one dose of treatment were included in the toxicity assessment. For the toxicity analysis, the data indicating the worst toxicity for each patient from all of the chemotherapy cycles were used.

\section{Statistical analysis}

According to Simon's optimal two-stage design [16], 38 patients were required for enrollment to test the null hypothesis that the true ORR is $20 \%$ versus the alternative hypothesis that the true ORR is at least $45 \%$, at a significance level of 0.05 with a power of $90 \%$. If five or more responses were observed among 16 patients in the first stage, the study was continued with 22 additional patients included. As the drop-out rate was assumed to be $10 \%$, the number of patients necessary for recruitment into the study was calculated to be 43 .

The intention-to-treat (ITT) population included all of the patients, and the per-protocol (PP) population excluded those patients who received treatment for less than 6 weeks for reasons other than disease progression or death or who received less than $50 \%$ of the anticipated treatment during the first 6 weeks of the trial.

The response and toxicity data were described using simple descriptive statistics. PFS was calculated from the first day of treatment until the first day of documented disease progression or death from any cause. PFS was censored at the date of the last follow-up visit for the patients who were still alive and had no documented disease progression. OS was calculated from the first day of treatment until the day of death. The response duration was defined as the time from the first day of a documented partial or complete response until the documented date of disease progression. PFS and OS were estimated using the Kaplan-Meier method.

\section{Results}

\section{Patient characteristics}

From September 2007 to May 2009, 44 patients who met the inclusion criteria were enrolled in this study. The demographic and pathologic characteristics of the patients are described in Table 1. The median age was 54.5 years 
(range 25-69 years). Thirty-two patients $(72.7 \%)$ were male, and only one patient $(2.3 \%)$ had an ECOG PS of 2. All patients had metastatic disease. Thirty-four patients $(77.3 \%)$ had an initial diagnosis of metastatic disease, and ten patients $(22.7 \%)$ had recurrent gastric cancer after curative surgery. Twenty-seven patients $(61.4 \%)$ had poorly differentiated cancers, and the commonest metastatic sites were distant lymph nodes $(80.1 \%)$, the peritoneum $(43.2 \%)$, and the liver $(27.3 \%)$. The median number of metastatic organs was 2 (range 1-4).

Table 1 Patient characteristics $(n=44)$

\begin{tabular}{|c|c|}
\hline Characteristics & No. of patients \\
\hline \multicolumn{2}{|l|}{ Gender } \\
\hline Male & $32(72.7 \%)$ \\
\hline Female & $12(27.3 \%)$ \\
\hline \multicolumn{2}{|l|}{ Performance status (ECOG) } \\
\hline 0 & $14(31.8 \%)$ \\
\hline 1 & $29(65.9 \%)$ \\
\hline 2 & $1(2.3 \%)$ \\
\hline \multicolumn{2}{|c|}{ Location of primary tumor site } \\
\hline Cardia & $3(6.8 \%)$ \\
\hline Fundus & $1(2.3 \%)$ \\
\hline Body & $15(34.1 \%)$ \\
\hline Antrum or pylorus & $12(27.3 \%)$ \\
\hline Diffuse & $12(27.3 \%)$ \\
\hline Unknown & $1(2.3 \%)$ \\
\hline \multicolumn{2}{|l|}{ Histology } \\
\hline Well differentiated & $5(11.4 \%)$ \\
\hline Moderately differentiated & $12(27.3 \%)$ \\
\hline Poorly differentiated & $27(61.4 \%)$ \\
\hline \multicolumn{2}{|l|}{ Prior surgery } \\
\hline Curative & $10(22.7 \%)$ \\
\hline Palliative & $2(4.5 \%)$ \\
\hline No surgery & $32(72.7 \%)$ \\
\hline \multicolumn{2}{|l|}{ Metastatic site } \\
\hline Liver & $12(27.3 \%)$ \\
\hline Lung & $2(4.5 \%)$ \\
\hline Lymph node & $37(80.1 \%)$ \\
\hline Peritoneum & $19(43.2 \%)$ \\
\hline Ovary & $2(4.5 \%)$ \\
\hline Others & $9(20.5 \%)$ \\
\hline \multicolumn{2}{|l|}{ No. of metastatic sites } \\
\hline 1 & $15(34.1 \%)$ \\
\hline 2 & $19(43.2 \%)$ \\
\hline$\geq 3$ & $10(22.7 \%)$ \\
\hline
\end{tabular}

The median age of the patients was 54.5 years (range 25-69 years). ECOG Eastern Cooperative Oncology Group

\section{Treatment delivery}

In total, 340 treatment cycles were administered to 44 patients, with a median of eight cycles (range 1-36 cycles) per patient. One patient had to discontinue use of docetaxel because of grade 4 neutropenia and received oxaliplatin and S-1 doublet therapy for the remaining eight of $13 \mathrm{cy}-$ cles. Five patients received the doublet of docetaxel and S-1 after experiencing grade 3 peripheral neuropathy. Two patients did not complete the first cycle of chemotherapy: one patient died suddenly of abdominal hemorrhage due to hepatic tumor rupture caused by disease progression, and the other patient was lost to follow-up. Thirty-one patients $(70.5 \%)$ received six or more cycles of chemotherapy. Twenty-seven patients $(61.4 \%)$ required dose reductions or delays. The mean relative dose intensities (ratio of the dose received to the dose planned) of docetaxel, oxaliplatin, and S-1 for all of the cycles administered were 0.89 [95\% confidence interval (CI) 0.86-0.92], 0.89 (95\% CI 0.86-0.92), and 0.88 (95\% CI 0.85-0.91), respectively. The median relative dose intensities of docetaxel, oxaliplatin, and S-1 for all of the cycles administered were 0.92 (95\% CI 0.89-0.95), 0.92 (95\% CI 0.90-0.94), and 0.92 (95\% CI 0.88-0.95), respectively. The dose reductions and delays during the first eight treatment cycles (total of 264 cycles in 42 patients) were as follows: the doses of docetaxel, oxaliplatin, and S-1 were reduced in 24 cycles $(9.1 \%), 26$ cycles $(9.8 \%)$, and 38 cycles $(14.4 \%)$, respectively; 42 cycles $(15.9 \%)$ were delayed. The commonest reasons for dose reduction and delay were grade $3 / 4$ neutropenia and leukopenia.

\section{Safety}

Safety was assessed in 43 patients on the basis of 339 cycles; one patient who was lost to follow-up after receiving docetaxel and oxaliplatin on day 1 of the first cycle, was excluded. One patient died suddenly of abdominal hemorrhage due to hepatic tumor rupture on day 3 of the first cycle. The adverse events are listed in Table 2. The commonest grade 3/4 hematologic toxicities were neutropenia $(37.2 \%)$ and leukopenia $(27.9 \%)$. Febrile neutropenia developed in six patients $(14.0 \%)$, all of whom recovered without complication. Nonhematologic toxicities were usually mild and manageable. Grade 3 toxicities with a frequency of $5 \%$ or more included peripheral neuropathy and deep vein thrombosis. Grade 3 peripheral neuropathy was observed in six patients (14.0\%), five of whom continued with the study regimen without receiving oxaliplatin. 
Table 2 Observed adverse events according to the number of patients and cycles

\begin{tabular}{|c|c|c|c|c|c|c|c|c|c|c|}
\hline \multirow[t]{3}{*}{ Event } & \multicolumn{5}{|c|}{ Patients $(n=43)$} & \multicolumn{5}{|c|}{ Cycles $(n=339)$} \\
\hline & \multicolumn{5}{|c|}{ NCI-CTCAE, version 3.0} & \multicolumn{5}{|c|}{ NCI-CTCAE, version 3.0} \\
\hline & 1 & 2 & 3 & 4 & $3 / 4(\%)$ & 1 & 2 & 3 & 4 & $3 / 4(\%)$ \\
\hline Leukopenia & 8 & 8 & 7 & 5 & 27.9 & 38 & 15 & 12 & 5 & 5.0 \\
\hline Neutropenia & 5 & 11 & 6 & 10 & 37.2 & 27 & 22 & 9 & 13 & 6.5 \\
\hline Anemia & 18 & 19 & 4 & 1 & 11.6 & 208 & 94 & 6 & 1 & 2.1 \\
\hline Thrombocytopenia & 13 & 1 & 1 & 1 & 4.7 & 41 & 2 & 2 & 1 & 0.9 \\
\hline Febrile neutropenia & - & - & 5 & 1 & 14.0 & - & - & 6 & 1 & 2.1 \\
\hline Anorexia & 23 & 15 & 2 & 0 & 4.7 & 178 & 27 & 3 & 0 & 0.9 \\
\hline Nausea & 26 & 10 & 0 & 0 & 0 & 120 & 16 & 0 & 0 & 0 \\
\hline Vomiting & 16 & 7 & 0 & 0 & 0 & 40 & 11 & 0 & 0 & 0 \\
\hline Diarrhea & 17 & 6 & 2 & 0 & 4.7 & 46 & 14 & 2 & 0 & 0.6 \\
\hline Stomatitis & 20 & 5 & 2 & 0 & 4.7 & 74 & 11 & 2 & 0 & 0.6 \\
\hline Hyperbilirubinemia & 8 & 6 & 0 & 0 & 0 & 24 & 9 & 0 & 0 & 0 \\
\hline AST/ALT & 16 & 7 & 0 & 0 & 0 & 72 & 9 & 0 & 0 & 0 \\
\hline Peripheral neuropathy & 22 & 10 & 6 & 0 & 14.0 & 167 & 64 & 7 & 0 & 2.1 \\
\hline Hand-foot syndrome & 16 & 6 & 0 & 0 & 0 & 73 & 19 & 0 & 0 & 0 \\
\hline Asthenia & 26 & 10 & 1 & 0 & 2.3 & 191 & 20 & 1 & 0 & 0.3 \\
\hline Deep vein thrombosis & 0 & 0 & 4 & 0 & 9.3 & 0 & 0 & 8 & 0 & 2.4 \\
\hline Hemorrhage & 0 & 0 & 0 & 1 & 2.3 & 0 & 0 & 0 & 1 & 0.3 \\
\hline
\end{tabular}

$A L T$ alanine aminotransferase, AST aspartate aminotransferase, NCI-CTCAE National Cancer Institute Common Terminology Criteria for Adverse Events

\section{Efficacy}

Of the 44 patients, 40 were eligible for response evaluation. Four patients were not available for response evaluation: three were lost to follow-up, and one died of rupture of a metastatic liver mass before response evaluation. Tumor responses are summarized in Table 3. There were three complete responses, 21 partial responses, 15 cases of stable disease, and one case of disease progression. The confirmed ORR was $54.5 \%$ (95\% CI 40.1-68.3\%) in the ITT population and $60.0 \%$ (95\% CI 44.6-73.7\%) in the PP population. The disease control rate was $88.6 \%(95 \% \mathrm{CI}$ 76.0-95.1\%) in the ITT population and $97.5 \%$ (95\% CI 87.1-99.5\%) in the PP population. The median time to response was 1.4 months (95\% CI 1.3-1.5 months), and the median duration of response was 7.4 months $(95 \% \mathrm{CI}$ 3.8-11.0 months).

At the time of analysis, 16 patients $(36.4 \%)$ were still alive with a median follow-up duration of 12.9 months (95\% CI 9.6-16.3 months). The median PFS was 7.6 months (95\% CI 6.2-9.0 months), and the median OS was 12.0 months (95\% CI 6.9-17.2 months), with a 2-year survival rate of $38.6 \%$ (95\% CI $24.7-54.5 \%$ ) in the ITT population as shown in Fig. 1.

Among the various clinical parameters, the response to treatment was the only prognostic factor that was identified in univariate and multivariate analyses.
Table 3 Treatment efficacy (assessed by an independent response review committee)

\begin{tabular}{lcll}
\hline Response & $\begin{array}{l}\text { No. of } \\
\text { patients }\end{array}$ & $\begin{array}{l}\text { Percentage of } \\
\text { patients }\end{array}$ & $\begin{array}{l}\text { 95\% CI } \\
(\%)\end{array}$ \\
\hline Complete response & 3 & & \\
Partial response & 21 & & \\
Stable disease & 15 & & \\
Progressive disease & 1 & & \\
Not evaluable & 4 & & $40.1-68.3$ \\
Intention-to-treat population & & $76.0-95.1$ \\
$\quad$ Overall response & & 54.5 & \\
$\quad$ Disease control & & 88.6 & $44.6-73.7$ \\
Per-protocol population & & & $87.1-99.5$ \\
$\quad$ Overall response & & 60.0 & \\
Disease control & & 97.5 & \\
\hline
\end{tabular}

CI confidence interval

\section{Discussion}

To the best of our knowledge, this is the first phase II study to evaluate the efficacy and feasibility of the DOS regimen as a palliative chemotherapy in patients with metastatic gastric cancer. The primary outcome was ORR, and the secondary outcomes were safety, PFS, and OS. The observed ORR was $54.5 \%$ in the ITT population, with a 


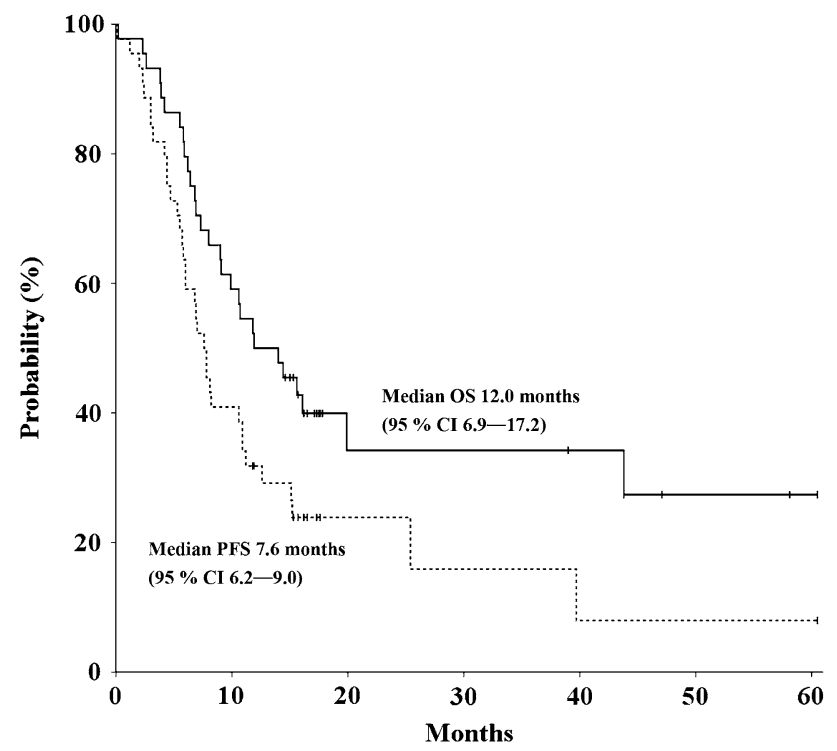

Fig. 1 Kaplan-Meier estimates of progression-free survival (PFS) and overall survival $(O S)$. $C I$ confidence interval

median PFS of 7.6 months and a median OS of 12.0 months. Although this was a phase II study, these efficacy results were favorably comparable to those obtained using DCF as first-line chemotherapy for AGC [4]. That study reported an ORR of $37 \%$, a median TTP of 5.6 months, and a median OS of 9.2 months. The DCF regimen of the V325 study consisted of docetaxel at $75 \mathrm{mg} / \mathrm{m}^{2}$ and cisplatin at $75 \mathrm{mg} / \mathrm{m}^{2}$ on day 1 plus $5-\mathrm{FU}$ at $750 \mathrm{mg} / \mathrm{m}^{2}$ on days $1-5$, every 3 weeks. The dose of docetaxel was lower in this DOS regimen than in the DCF regimen without sacrificing treatment efficacy. Moreover, the low dose intensity was translated into low incidences of grade 3/4 hematologic toxicities. Grade 3/4 neutropenia and febrile neutropenia were observed in 38.1 and $14.3 \%$ of patients in this study, whereas the DCF regimen resulted in much higher occurrences of 82 and $29 \%$, respectively. Given that the major concern of three-drug regimens is severe toxicity, these findings suggest an advantage of the DOS regimen. Recently, a phase II study of neoadjuvant DOS chemotherapy was reported [17]. Although most patients had stage II or stage III disease, the ORR was 53.8 and the disease control rate was $100 \%$ after three cycles of DOS therapy. Grade 3/4 neutropenia and febrile neutropenia were observed in 34.1 and $9.8 \%$ of patients, respectively. These findings of efficacy and toxicity were comparable to those of the current study, and provide support for the feasibility of the DOS regimen.

Capecitabine in combination with docetaxel and oxaliplatin (DOX) is another regimen investigated for the treatment of gastric cancer. A 3-week DOX regimen consisting of docetaxel at $60 \mathrm{mg} / \mathrm{m}^{2}$ and oxaliplatin at $100 \mathrm{mg} /$ $\mathrm{m}^{2}$ on day 1 and continuous orally administered capecitabine at $1000 \mathrm{mg} / \mathrm{m}^{2}$ daily yielded an ORR of $52.1 \%$, a median PFS of 6.9 months, and a median OS of 12.6 months [18]. When docetaxel at $50 \mathrm{mg} / \mathrm{m}^{2}$ and oxaliplatin at $75 \mathrm{mg} / \mathrm{m}^{2}$ were administered intravenously on day 1 , and capecitabine at $2750 \mathrm{mg} / \mathrm{m}^{2}$ was administered orally on days 1-7 every 2 weeks, the ORR, median TTP, and median OS were $59 \%, 10.0$ months, and 18.0 months, respectively [19]. Considering also our study, docetaxelcontaining triplet regimens demonstrated better treatment outcomes compared with those for doublets including platinum and fluoropyrimidine. Among docetaxel-containing triplets, the treatment outcomes for the DOS regimen in the current study are comparable to those for the DOX regimens described above. However, the DOS regimen showed more favorable toxicity profiles in terms of hand-foot skin (HFS) reaction, one of the commonest toxicities with capecitabine to limit the daily activity of patients. The frequency of grade 3 or higher HFS reactions was much lower with the DOS regimen compared with the DOX regimens (0 \% vs 6-15\%).

Recently, a phase III study comparing oxaliplatin plus S-1 (SOX) with cisplatin plus S-1 in chemotherapy-naive patients with AGC reported that SOX was as effective as cisplatin plus S-1, with a favorable safety profile [20]. Patients assigned to the SOX group received oxaliplatin at $100 \mathrm{mg} / \mathrm{m}^{2}$ on day 1 , and $\mathrm{S}-1$ at $80 \mathrm{mg} / \mathrm{m}^{2}$ for 2 weeks, every 3 weeks. The SOX regimen resulted in an ORR of $55.7 \%$, median PFS of 5.5 months, and median OS of 14.1 months. Grade 3/4 neutropenia and febrile neutropenia were seen in 19.5 and $0.9 \%$ of SOX patients, respectively. The ORR for DOS was similar to that for SOX, however, addition of docetaxel seemed to cause more hematologic toxicities. Considering these results, comparative clinical trials with DOS and SOX seem to be warranted to identify the role of docetaxel in combination with SOX.

The treatment was generally well tolerated by most patients. The commonest grade $3 / 4$ adverse event was neutropenia (37.2\% of all patients), and the second commonest grade $3 / 4$ adverse event was leukopenia (27.9\%). Six patients $(14.0 \%)$ experienced febrile neutropenia. Although peripheral neuropathy of any grade was observed in most patients $(88.4 \%)$, grade 3 peripheral neuropathy was observed in six of 11 patients who received nine or more cycles of chemotherapy. On the other hand, only grade 1 and grade 2 HFS reactions were observed. Few grade $3 / 4$ nonhematologic toxicities were observed, including anorexia, diarrhea, stomatitis, and deep vein thrombosis. Possible explanations for the favorable toxicity profiles include the younger patient population, a good ECOG PS, and strict adherence to the guideline for dose reduction or delay. The median age in our study was 54.5 years, and all patients except one had a good ECOG 
PS (0 or 1$)$. In addition, treatment was interrupted in patients with grade 2 or higher toxicity. Dose modification or delay conducted strictly according to the study protocol might have reduced the chance of developing severer (grade 3/4) toxicities in subsequent cycles.

As expected, the administration of the DOS regimen was convenient for the patients. Unlike the 5-FU-based regimens that require intravenous infusion of 5 -FU for 2 or 5 days, the DOS regimen requires only a $3 \mathrm{~h}$ infusion of docetaxel and oxaliplatin and oral administration of S-1. Most patients in this study had a good PS. Although it was deemed an unintended consequence, it might overestimate the efficacy and safety of the DOS regimen. So, a larger study in a more heterogeneous patient group with the DOS regimen is needed.

In conclusion, the DOS regiment is effective, relatively well tolerated, and convenient. It seems to have potential to be a reasonable therapeutic strategy. Therefore, a larger study is warranted to confirm the efficacy and safety of the DOS regimen in the treatment of metastatic gastric cancer.

Acknowledgments This work was supported by Hallym University Medical Center. Docetaxel and oxaliplatin were provided by SanofiAventis Korea, and S-1 was provided by Jeil Pharmaceutical.

\section{References}

1. Ferlay J, Soerjomataram I, Ervik M, Dikshit R, Eser S, Mathers C, et al. GLOBOCAN 2012 v1.0, Cancer Incidence and Mortality Worldwide: IARC CancerBase No. 11 [Internet]. Lyon, France: International Agency for Research on Cancer; 2013. Available from http://globocan.iarc.fr. Accessed 28 Jan 2015.

2. Yoo CH, Noh SH, Shin DW, Choi SH, Min JS. Recurrence following curative resection for gastric carcinoma. Br J Surg. 2000;87:236-42.

3. Wagner AD, Grothe W, Haerting J, Kleber G, Grothey A, Fleig WE. Chemotherapy in advanced gastric cancer: a systematic review and meta-analysis based on aggregate data. J Clin Oncol. 2006;24:2903-9.

4. Van Cutsem E, Moiseyenko VM, Tjulandin S, Majlis A, Constenla M, Boni C, et al. Phase III study of docetaxel and cisplatin plus fluorouracil compared with cisplatin and fluorouracil as firstline therapy for advanced gastric cancer: a report of the V325 study group. J Clin Oncol. 2006;24:4991-7.

5. Sulkes A, Smyth J, Sessa C, Dirix LY, Vermorken JB, Kaye S, et al. Docetaxel (Taxotere) in advanced gastric cancer: results of a phase II clinical trial. EORTC early clinical trials group. Br J Cancer. 1994;70:380-3.

6. Mavroudis D, Kourousis C, Androulakis N, Kalbakis K, Agelaki $\mathrm{S}$, Kakolyris S, et al. Frontline treatment of advanced gastric cancer with docetaxel and granulocyte colony-stimulating factor (G-CSF): a phase II trial. Am J Clin Oncol. 2000;23:341-4.
7. Bang YJ, Kang WK, Kang YK, Kim HC, Jacques C, Zuber E, et al. Docetaxel $75 \mathrm{mg} / \mathrm{m}^{2}$ is active and well tolerated in patients with metastatic or recurrent gastric cancer: a phase II trial. Jpn J Clin Oncol. 2002;32:248-54.

8. Ridwelski K, Gebauer T, Fahlke J, Kröning H, Kettner E, Meyer $\mathrm{F}$, et al. Combination chemotherapy with docetaxel and cisplatin for locally advanced and metastatic gastric cancer. Ann Oncol. 2001;12:47-51.

9. Roth AD, Fazio N, Stupp R, Falk S, Bernhard J, Saletti P, et al. Docetaxel, cisplatin, and fluorouracil; docetaxel and cisplatin; and epirubicin, cisplatin, and fluorouracil as systemic treatment for advanced gastric carcinoma: a randomized phase II trial of the Swiss group for clinical cancer research. J Clin Oncol. 2007;25:3217-23.

10. Al-Batran SE, Hartmann JT, Probst S, Schmalenberg H, Hollerbach S, Hofheinz R, et al. Phase III trial in metastatic gastroesophageal adenocarcinoma with fluorouracil, leucovorin plus either oxaliplatin or cisplatin: a study of the Arbeitsgemeinschaft Internistische Onkologie. J Clin Oncol. 2008;26:1435-42.

11. Cunningham D, Starling N, Rao S, Iveson T, Nicolson M, Coxon $\mathrm{F}$, et al. Capecitabine and oxaliplatin for advanced esophagogastric cancer. N Engl J Med. 2008;358:36-46.

12. Boku N, Yamamoto S, Fukuda H, Shirao K, Sawaki A, et al. Fluorouracil versus combination of irinotecan plus cisplatin versus S-1 in metastatic gastric cancer: a randomised phase 3 study. Lancet Oncol. 2009;10:1063-9.

13. Ajani JA, Rodriguez W, Bodoky G, Moiseyenko V, Lichinitser M, Gorbunova V, et al. Multicenter phase III comparison of cisplatin/S-1 with cisplatin/infusional fluorouracil in advanced gastric or gastroesophageal adenocarcinoma study: the FLAGS trial. J Clin Oncol. 2010;28:1547-53.

14. Zang DY, Yang DH, Kim MJ, Jang KM, Hwang SW, Yoo KS, et al. Dose-finding study of docetaxel, oxaliplatin, and S-1 for patients with advanced gastric cancer. Cancer Chemother Pharmacol. 2009;64:877-83.

15. Therasse P, Arbuck SG, Eisenhauer EA, Wanders J, Kaplan RS, Rubinstein L, et al. New guidelines to evaluate the response to treatment in solid tumors. J Natl Cancer Inst. 2000;92:205-16.

16. Simon R. Optimal two-stage designs for phase II clinical trials. Control Clin Trials. 1989;10:1-10.

17. Park I, Ryu MH, Choi YH, Kang HJ, Yook JH, Park YS, et al. A phase II study of neoadjuvant docetaxel, oxaliplatin, and S-1 (DOS) chemotherapy followed by surgery and adjuvant S-1 chemotherapy in potentially resectable gastric or gastroesophageal junction adenocarcinoma. Cancer Chemother Pharmacol. 2013;72:815-23.

18. Di Lauro L, Vici P, Belli F, Fattoruso SI, Arena MG, Pizzuti L, et al. Docetaxel, oxaliplatin, and capecitabine combination chemotherapy for metastatic gastric cancer. Gastric Cancer. 2014;17:718-24.

19. Amarantidis K, Xenidis N, Chelis L, Chamalidou E, Dimopoulos $\mathrm{P}$, Michailidis $\mathrm{P}$, et al. Docetaxel plus oxaliplatin in combination with capecitabine as first-line treatment for advanced gastric cancer. Oncology. 2011;80:359-65.

20. Yamada Y, Higuchi K, Nishikawa K, Gotoh M, Fuse N, Sugimoto N, et al. Phase III study comparing oxaliplatin plus S-1 with cisplatin plus $\mathrm{S}-1$ in chemotherapy-naïve patients with advanced gastric cancer. Ann Oncol. 2015;26:141-8. 